\title{
3-D SIMULATION OF DOUBLE-BAR PLUSH FABRICS WITH JACQUARD PATTERNS
}

\author{
Aijun Zhang', , Xinxin Li', Gaoming Jiang ${ }^{1,2}$, Zhijia Dong ${ }^{1,3}$, Honglian Cong ${ }^{1,2, *}$ \\ ${ }^{1}$ Engineering Research Center for Knitting Technology, Ministry of Education, Jiangnan University, Wuxi, China; \\ ${ }^{2}$ International Joint Research Laboratory for Noval Knitting Structural Materials at Jiangnan University, Wuxi, China; \\ ${ }^{3}$ Key Laboratory of Eco-textiles, Ministry of Education, Wuxi, China; \\ *Corresponding Authors: zhangaijun@live.com; cong-wkrc@163.com
}

\section{Abstract:}

A realistic computerized simulation of double-bar plush fabrics can result in a time-saving development process with high quality. Based on basic analysis of jacquard principles, a fast 3-D simulation method of warp-knitted plush fabrics is proposed by using a geometry shader on GPU. Firstly, pile areas and non-pile areas are identified according to the jacquard design graphs and chain notations. According to the directions of observation and raised pile, two layered chips are formed in the geometry shader with an approach of multi-layered textures. To ensure that the simulated piles resemble the real ones, the directions of the piles are randomized with the Perlin noise method. One pile is generated along its length with numerous layers in the plush fabric model. Simulation results of piles on both the technical face and technical back are obtained via the model built above, which is confirmed with practicability and efficiency. This $3 D$ simulation approach improves the visualization appearance of piles just as they are actually raised.

\section{Keywords:}

warp knitting; plush fabrics; fabric simulation; 3D modeling; layered texture; geometry shader

\section{Introduction}

Double-bar plush fabrics with jacquard patterns are produced on warp knitting double-bar raschel machines and widely used in various products, such as blankets, clothing and lining, man-made furs, bedclothes and toy fabrics. Traditional ways to form plush pattern effects include selecting colorful yarns, printing and embossing. With an advancement of piezo jacquard technology, the double-bar raschel machine provides a new approach to generating decorative jacquard-patterned plush fabrics. Based on chain notations of jacquard bars and color definition of jacquard grids, a jacquard pattern bitmap is transferred into machine data to control fabrication of patterned plush fabrics. Due to complicated techniques of jacquard patterning and an unpredictability of design results, the fabrication of patterned plush fabrics is quite time-consuming and uneconomical, which reveals an urgent need for real-time CAD simulation of the appearance of fabrics to help craftsman check design results in the drafting process.

Unlike other warp-knitted fabrics, a plush fabric is knitted by jointly using the ground bars to form ground structures and using the jacquard bars for patterned piles. In pile areas, ground loops are covered by raised pile. Simulation of the plush fabrics involves building models of the ground stitch loops, the jacquard-patterned pile as well as the spatial coverage inspection. Cong ${ }^{[1]}$ simulated pile fabrics through texture synthesis. This method created realistic results by taking images from original real fabrics, resulting in a need to collect large quantities of texture images and a problem that the form of piles is unchangeable. Le ${ }^{[2]}$ converted the image pixels of piles into particles, and then simulated the pile effects by using the radial fuzzy algorithm. In details, several pixel values were selected from a line connecting all the pixel points and its fuzzy center point in the drafted pattern. The mean of the extracted pixel values was computed as the new pixel value of this point. Xiong ${ }^{[3]}$ simulated the pile effects with a line method by describing the pile yarn as a line segment with a gradually changing color. The algorithms mentioned above are focused on the 2-D images or patterns and need to be further improved in terms of 3-D simulation effects. The methods for 3-D simulation of pile include the geometric modeling and texture generating. Csuri, ${ }^{[4]}$ Neulander, ${ }^{[5]}$ Sadeghi, ${ }^{[6]}$ and Yan $^{[7]}$ drew the animal furs very clearly with the geometric method, but it was time-consuming and mostly used for offline rendering for film production and animation. Kajiya ${ }^{[8]}$ plotted furs with three-dimensional texture generating method, and the plotted fur was very real and soft, but the early methods were not efficient in simulating knitting products. Lengye ${ }^{[9,10]}$ proposed a method of drawing the plush with texture layers. This method firstly generated a tuft of plush via the particle system and then collected numerous sampling textures on different layers to map them on a set of meshes to form plush effects. It shows advantages in both visualization results and rendering efficiency, thus making it the basis of further researches. Yang ${ }^{[11]}$ replenished Lengyel's method with a control algorithm of vector field to realize the simulating interacted piles. It redefined the shift vectors of vertexes on meshes to build piles with different leaning directions. Different from Lengyel's way, which generated an overall fin, Tang ${ }^{[12]}$ divided the fin into multiplied layers and generated each layer according to its mesh shift to guarantee that it adapts to dynamic forms of piles. Wong ${ }^{[13]}$ realized the 3D simulation of snow scene with multi-layered textures method. Lee ${ }^{[14]}$ presented a model in which normal vectors of meshes were affected 
by gravity, wind and customer's interactions. Meanwhile, it improved the randomness of piles' appearances by displaying both straight piles and lean piles, which was applied into simulation of fur garments. Zhang ${ }^{[15]}$ applied Lengyel's method into the simulation of single-jacquard patterned plush fabrics. $\mathrm{He}$ generated texture layers by employing restrictions to random vertexes, which enabled the piles to have minor offset in a small scale.

Lengyel's model and models based on that guaranteed both simulation results and computation efficiency. Compared with 2D simulation models, more realistic effects are shown in the $3 \mathrm{D}$ simulation models with the multi-layered textures method. Compared with traditional 3D geometrical methods, the way to generate semi-transparent textures presented by Lengyel had much more efficiency to meet the real-time requirements of CAD programs. Nevertheless, this model took a mesh of piles as the basic unit, which means it is impossible to adjust the stretching direction of one single pile. Moreover, all piles on a mesh had the same reflected effects due to the same illumination model. With the development of GPU (Graphics Processing Unit), the geometry shader became a part of programmable pipelines, breaking bottlenecks of GPU method by using parallel computing to efficiently extend the geometrical models. This paper realized the real-time 3D simulation of jacquard-patterned plush fabrics by adopting the multi-layered textures method and using the geometry shader to generate the piles appearances. Since the model takes each single pile as a simulated unit, the effects of each one's stretching direction and lighting results can be instantly obtained, resulting in better application in simulating plush fabrics with long piles or lean piles.

\section{Knitting and jacquard principles}

The warp-knitting double-bar raschel machine for producing jacquard-patterned plush fabrics has two needle beds, which are placed back to back and simultaneously knit 3D fabrics with two layers. ${ }^{[16]}$ RDJ5/1P, a typical machine for producing jacquard-patterned plush fabrics, is equipped with 6 guided bars and the regular structure is shown in Table 1.

GB1 and GB2 make lapping movements at the front needle bed to form stable structures of the front layer. GB4 and GB5, lapping at the back bed, form stable structures of the back layer. The jacquard pile guide bars JB3.1 and JB3.2 are half configured with guide needles interleaved to form a full jacquard guide bar as JB3. JB3 can work at both the front and back needle beds to generate the pile layer, which connects double layers. During the finishing process, the pile layer is cut through the middle and raised to form piles on the technical back. Piles of the technical face are pulled out on the gigging machine. In order to produce the patterned piles, it is necessary to control the Jacquard guide bars (JB3) during the knitting process. Most of jacquard fabrics are knitted by controlling the offset signals at underlapping to generate underlaps with different lengths and then to form the decorative patterns. But for the jacquard patterns on double-bar plush fabrics, they are knitted by controlling the offset signals at overlapping in order to generate the floating structures and then to form the nonpile areas. JB3 has four lapping movements in each course, that is, the underlapping and overlapping on the front needle bed, and the underlapping and overlapping on the back needle bed. Therefore, four displacement signals are controlled in one course for JB3.

Jacquard patterns are usually designed with jacquard colorful grids. Different colors on the jacquard patterns stand for different offset control signals. The front fabric and back fabric produced in this way have symmetrical patterns, and only a set of jacquard guide bars are available in the machine. Table 2 shows the relationship between the jacquard colors and chain notations used in the jacquard design.

As shown in Figure 1-a, the red color means that the jacquard needles make stitches at both front and back needle beds. Piles are generated after the jacquard underlaps are cut, which connect the front and back layers. The green color means that the jacquard needles make stitches at only one needle bed, forming ground fabrics as the non-pile heavy area, as shown in Figure 1-b and Figure 1-c. The white color means that the jacquard needles in this region only make floating structures on both the front and back needle beds. Therefore, the ground fabric is thin and has no piles, as shown in Figure 1-d. 3-D stereoscopic jacquard-patterned plush fabrics can be produced by a combination of the three colors.

Table 1. Chain notations of the double-bar jacquard-patterned plush fabrics

\begin{tabular}{|c|c|}
\hline Guided bars & Chain notations \\
\hline GB1 & $0-1-1-1 / 3-2-2-2 / /$ \\
\hline GB2 & $2-1-1-1 / 0-1-1-1 / /$ \\
\hline JB3.1+ JB3.2 & $1-0-1-0 / 1-0-1-0 / /$ \\
\hline GB4 & $1-1-2-1 / 1-1-0-1 / /$ \\
\hline GB5 & $1-1-0-1 / 2-2-3-2 / /$ \\
\hline
\end{tabular}

Table 2. Jacquard colors and control signals

\begin{tabular}{|c|c|c|c|c|}
\hline \multirow{2}{*}{ Basic chains } & Colors & Wales & Offset signals & Actual chains \\
\hline \multirow{3}{*}{$1-0-1-0 / /$} & Red & odd/even & HHHH & $1-0-1-0 / /$ \\
\cline { 2 - 5 } & \multirow{2}{*}{ Green } & odd & HHHT & $1-0-1-1 / /$ \\
\cline { 2 - 5 } & \multirow{2}{*}{ White } & even & HTHH & $1-1-1-0 / /$ \\
\cline { 2 - 5 } & & odd/even & HTHT & $1-1-1-1 / /$ \\
\hline
\end{tabular}




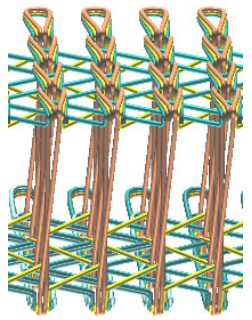

(a)
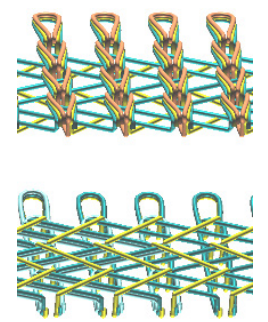

(b)

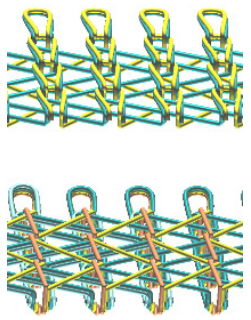

(c)
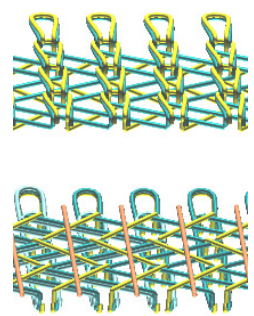

(d)

Figure 1. Fabric structures with different jacquard colors: (a) stitching at front and back bed; (b) stitching at front bed; (c) stitching at back bed; (d) do not stitch.

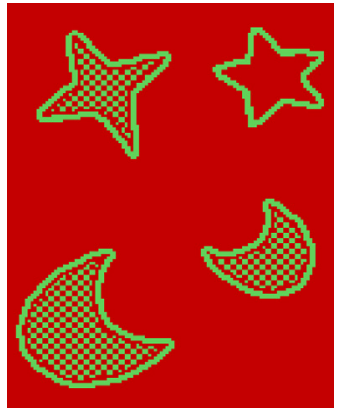

(a)

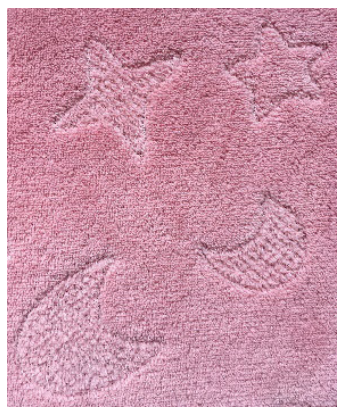

(b)

Figure 2. An example of the jacquard-patterned plush fabric. (a) the jacquard pattern; (b) the real fabric.

Figure 2-a shows an example of the double-bar plush fabrics with jacquard patterns. As it is well-known, the plush fabrics are mostly used to make blankets, and thus, they are expected to be thick and warm with large pile areas. Lines and points are often selected to form patterns.

\section{Simulation model of piles}

The piles at the front and back sides of plush fabrics have different forms. The fabric is cut into two pieces through the middle, and then sheared and carded to obtain a neat and upright pile at the technical back. However, the pile raised on technical face is fluffy and usually clustered together.

In the simulation model based on the layered texture, the 2-D form of pile chip needs to be defined first. Then the mapping relationship from 2-D chip to fabric model should be constructed. Finally, the chips are drawn at each layer of the fabric pile model, producing the pile simulation effect.

\section{$\underline{3.1 \text { Representation of piles }}$}

For the double-bar plush fabrics, it is assumed in this paper that a pile yarn is a soft, semi-transparent geometric figure generated by a circular cross-section scanning along a path from root to head.

Method of Lengyel partitioned the pile yarns into numerous layers and then rendered texture of each layer to generate a fluffy feeling pile effect. But when the observation direction is almost vertical to the pile yarn, interstice between layers is exposed. A similar idea is chosen in this paper, but two types of texture chips are used to draw an individual pile yarn with the geometry shader to avoid interstice.

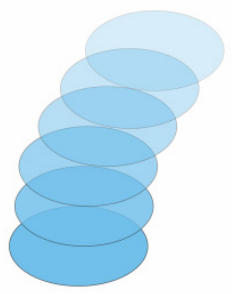

(a)

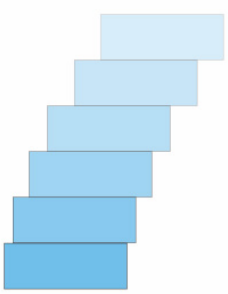

(b)
Figure 3. Texture chips. (a) horizontal and (b) vertical chips.

Figure 3-a shows the horizontal chips, each of which is vertical to the tangent line of the pile yarn. Figure 3-b shows the vertical chips, each of which is vertical to the observation direction. An appropriate texture chip is selected by judging the interstice between the layers in specific observation direction. When the observation is parallel to the tangent of the piles, a horizontal chip is preferred because of a tiny possibility of interstice, as shown in Figure 4-a. However, when the observation is nearly vertical to the pile, the vertical chips are better because an interstice is much easier to be formed if using horizontal chips (Figure 4-b, Figure 4-c).

The interstice between layers is related to the interval space between the layers $I$, radius $r$ of the pile yarns, and the included angle $\alpha$ between the observation direction and the pile yarns. The probability of interstice between the layers has a direct proportion with the distance between layers $/$ and the angle $\alpha$, but has an opposite proportion with $r$.

If $2 \cdot r \cdot \cos \alpha \geq l \cdot \sin \alpha$, then the projection length of the pile yarn diameter on the view plane is larger than the interval space between layers and the horizontal chip is preferred. If $2 \cdot r \cdot \cos \alpha<l \cdot \sin \alpha$, namely $\alpha>\tan ^{-1} \frac{2 \cdot r}{l}$, then the vertical chip is more suitable. 


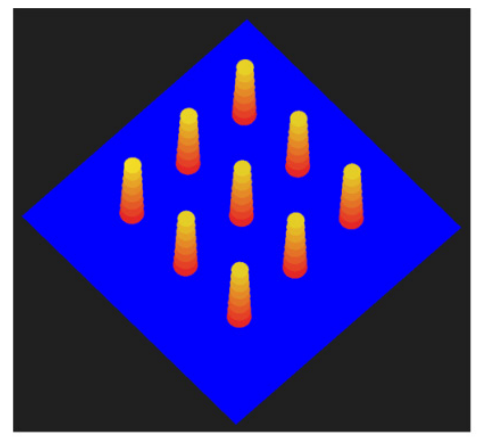

(a)

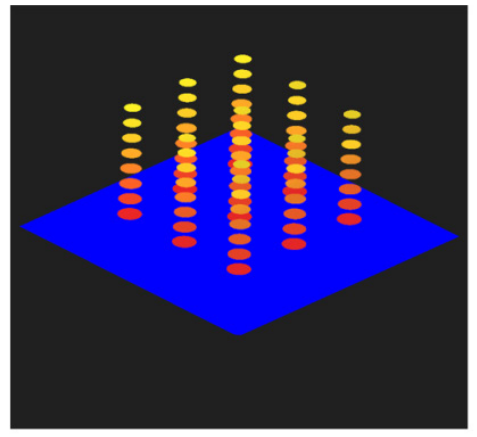

(b)

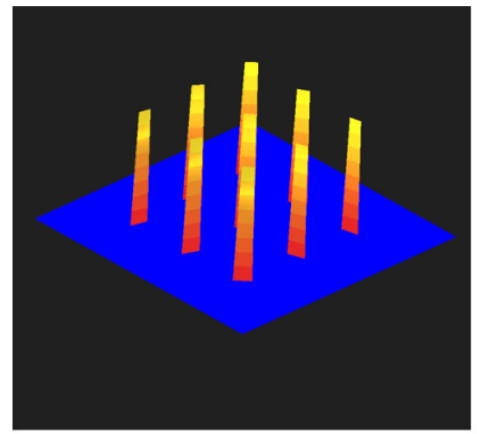

(c)

Figure 4. Selection of texture chips. (a) parallel observation with horizontal chips; vertical observation with (b)horizontal chips and (c) vertical chips.

\subsection{Introduction of Perlin fractal noise to the direction vector}

As the raised pile yarns are irregularly in random directions, a parameter of randomness is adopted to improve the realistic simulation effects of plush fabrics. White noise is random but it is uniformly distributed across the entire region so that the simulation result is not as natural as expected. Perlin noise method $^{[17]}$ is chosen in this paper to simulate the randomness of the pile yarn directions. The width and height of the noisecorrupted texture are defined as the same as the width and height of the pattern size of the plush fabrics. The value of the noise-corrupted texture represents the tangent direction of the pile yarns.

The noise-corrupted texture is generated in the following ways. First, random variables $\theta$ and $\lambda$ are generated. Assuming that the original direction of the pile yarns is the same as the $y$ axis in the 3-D coordinate system, $\theta$ denotes the included angle between the offset vector and the $y$ axis. It determines the offset of pile yarns and is usually set to a value less than $45^{\circ} . \lambda$ determines the offset direction of pile yarns, and it ranges from 0 to $360^{\circ}$. The offset location $(x, y, z)$ can be computed through $x=\sin \theta \cdot \cos \lambda, y=\cos \theta$, and $z=\sin \theta \cdot \sin \lambda . x, y$ and $z$ are then converted to the RGB color mode (namely the white noise texture) according to $R=\frac{x+1}{2} \times 255, G=\frac{y+1}{2} \times 255$ and $B=\frac{z+1}{2} \times 255$, which is shown in Figure 7-a. Figures 7-b, 7-c and 7-d show the sampling results of Figure $7-a$ under different sampling rates. The smaller the sampling rate is, the fewer minutiae are collected. Different sample figures are mixed to form the final Perlin fractal noise based on the following equation (Equation 1).

$$
P=\frac{\sum_{i=1}^{\mathrm{n}}\left(k_{i} \cdot P_{f(i)}\right)}{\sum_{i=1}^{\mathrm{n}} k_{i}}
$$

$P_{f(i)}$ denotes the sampling value at the sampling rate $f_{(i)}$, while $k_{i}$ denotes its corresponding proportion. $n$ denotes the total number of sampling times. Each of the sampling figures has different proportions when they are mixed. The figure that has fewer minutiae is allocated with a higher proportion. For example, there are the fewest minutiae in Figure 7-d but it has a larger proportion. And the larger proportion approximately determines the final result of Perlin fractal noise. By mixing

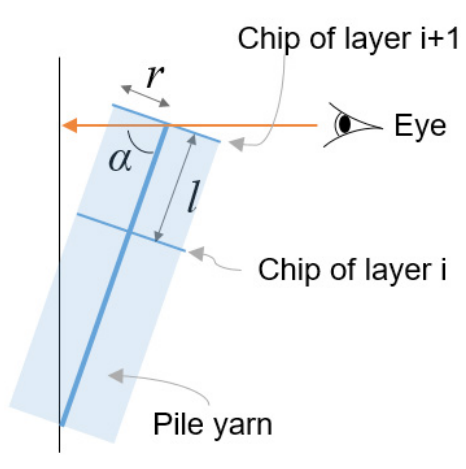

Figure 5. Projection of pile yarn on the view plane

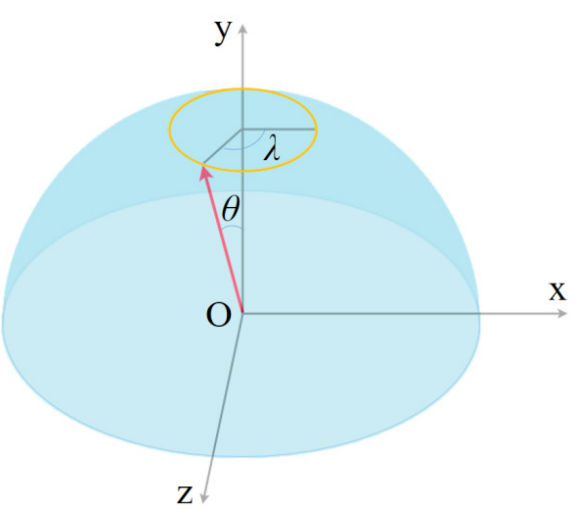

Figure 6. Generating random variables $\theta$ and $\lambda$
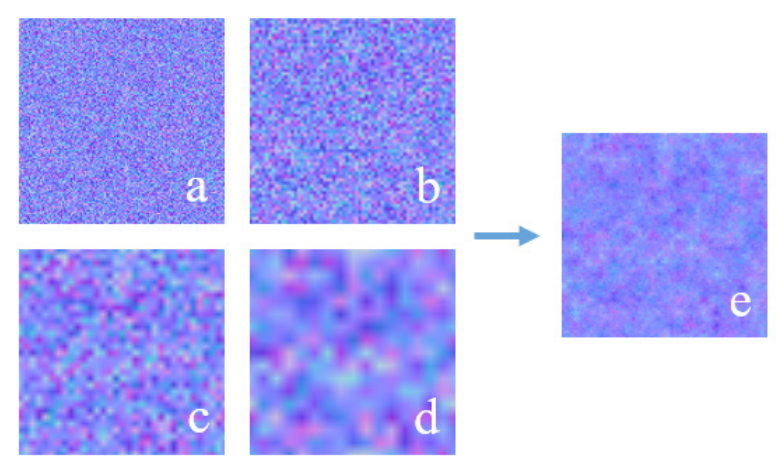

Figure 7. Combination of noise-corrupted texture. (a) sampling rate $=1$ (white noise); (b) sampling rate $=2$; (c) sampling rate $=4$; (d) sampling rate $=8 ;(e)$ Perlin fractal noise 
Figures 7-a, 7-b, 7-c and 7-d, we obtain the Perlin fractal noise texture in Figure 7-e.

Different noise effects are obtained by changing the parameters in Equation 1. If $n=2, f(1)<f(2), k_{1}<k_{2}$, the possible noise effects are listed in Table 3 . Revealed by the table, there is a direct relationship between the value of $f(i)$ and the unit size of noise texture. Bigger noise texture dots means a larger area of parallel piles. In contrast, a higher ratio of $k_{1} / k_{2}$ means more detailed textures of piles and generates a plush area with more messy piles. In specific application, if pile yarns are comparatively short and rigid, piles tend to keep a stable indirection; in such a circumstance, it's better to set bigger values of $f(1)$ and $f(2)$ but a lower ratio of $k_{1} / k_{2}$. While if pile yarns are long and softer, piles tend to lean in random directions, hence smaller values of $f(1)$ and $f(2)$ but a higher ratio of $k_{1} / k_{2}$ are needed.

The pile yarns without noises have a uniform direction and appearance, as shown in Figure 8-a, compared with realistic simulation results shown in Figure 8-b which is generated based on Figure 7-e with Perlin fractal noises.

\subsection{Cluster of pile yarns on technical face}

The piles on the technical face are shown as a cluster of pile yarns. In order to simulate this appearance, the fabric is equally partitioned into several regions, each of which has a cluster of piles centering the blue dots in Figure 9. Specially, the blue dots are randomly located. The root points of the surrounding pile yarns are represented with the gray dots in Figure 9. The space between gray points and surrounding blue points are computed and the nearest blue point is chosen as the leaning center of the surrounding pile yarns. When generating the pile effect on technical face, the head of surrounding pile yarns are leaning towards the central blue point to show the clustering effect.

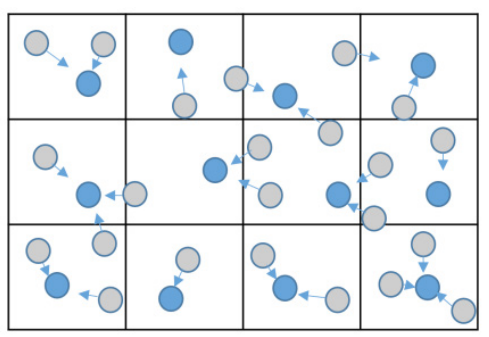

Figure 9. Clusters of pile yarns

\section{Drawing of Plush fabrics}

The geometry shader is introduced from DirectX 10. It allows GPU to dynamically generate and destroy data of geometric primitives, which means that the input primitives can be extended into other ones or be removed. Based on that, the point primitive that represents the pile root in GPU is transferred into the quadrilateral section with certain area as described in Section 3.1 and then finally is generated to be 3-D pile. This operation is performed by GPU, resulting in reduced computation on CPU and improving efficiency.

Table 3. Perlin fractal noise effects with different parameters

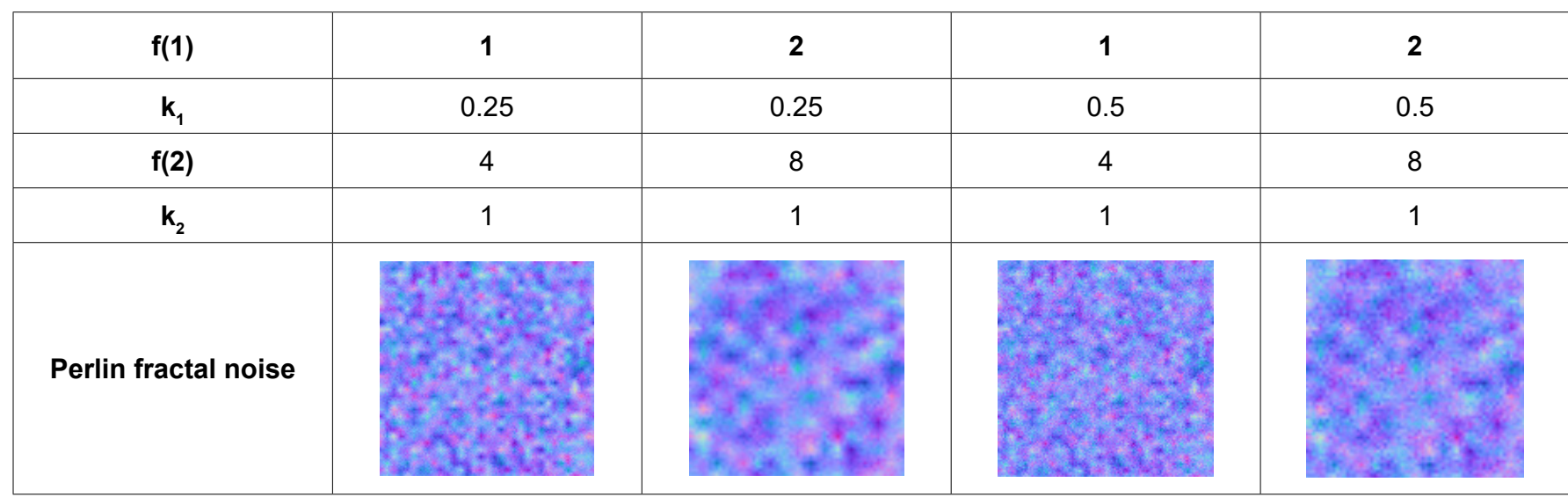

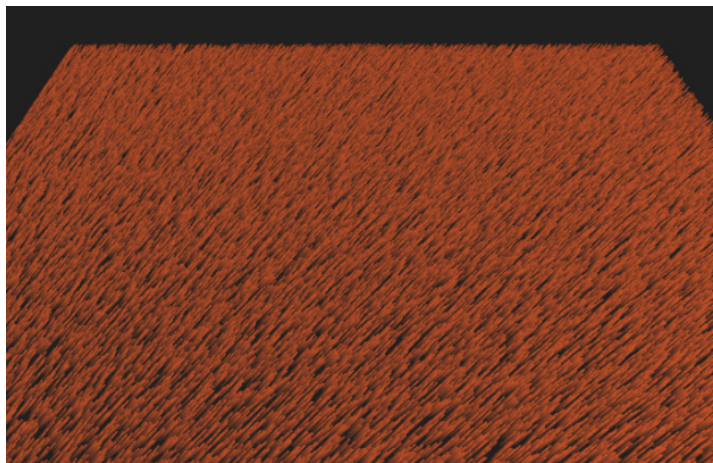

(a)

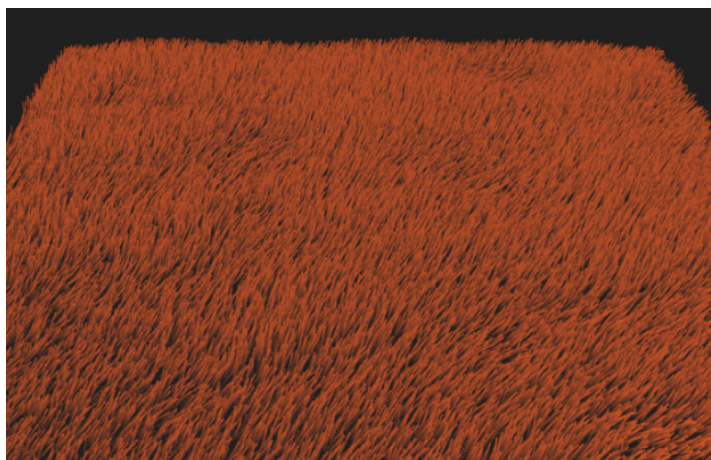

(b)

Figure 8. Simulation of pile yarns. (a) the result of uniform piles; (b) the result with Perlin fractal noises. 
The simulation flowchart is shown in Figure 10. The doublebar plush fabrics with jacquard patterns are comprised of ground loops, jacquard loops and piles. When simulating the fabric appearance, the loop curves are first modeled based on chain notations and threading cycle. After that, the 3D loop geometries are built by sweeping along the curves with sectional chips. Jacquard structures are modeled based on jacquard pattern bitmaps and jacquard grids definition (explained in the part of Knitting and jacquard principles). Locations of jacquard pile areas are decided by jacquard bitmaps, and the direction of jacquard pile is determined by the method in the part of Simulation model of piles. To generate 3D jacquard piles, the geometry shader extends the position point of pile into a quadrilateral based on the direction texture to generate a tiny segment of pile on each layer. The simulation result can be obtained by simultaneously rendering the ground loops, jacquard loops and piles to the screen.

Rendering is done in the above-mentioned method by using the vertex shader, geometry shader and fragment shader. The vertex shader needs no conversion and merely introduces the vertex into the geometry shader. Each primitive of vertex on the fabric calls the geometry shader once. The shape of pile texture clip, horizontal or vertical, is decided with the algorithm in Section 3.1. Both of them are represented with quadrilateral sections to be mapped with pile texture. The degree of transparency is smaller for the layers close to the root than the layers close to the head.

The color is computed based on the following equation (Equation 2) in the fragment shader:

$$
I_{i}=I_{a} K_{a}+I_{d} K_{d} \sqrt{1-(T \cdot L)^{2}}+I_{s} K_{s}{\sqrt{1-\left(T \cdot \frac{L+V}{|L+V|}\right)^{2}}}^{s}
$$

Where, $l_{i}$ denotes the total intensity of light on the number $i$ layer; $I_{a}, I_{d}$ and $I_{s}$ respectively denote the light intensities of the environment, diffuse reflection and specular reflection;
$K_{a}, K_{d}$ and $K_{s}$ denote the light coefficient of the environment, diffuse reflection coefficient and specular reflection coefficient; $T$ denotes the tangent direction of the pile; $L$ denotes the light direction; $V$ denotes the observation direction; $s$ denotes the reflection exponent.

The computed colors are blended using the following equation:

$$
I_{\text {new }}=I_{i} S_{i}+I_{\text {old }}\left(1-S_{i}\right)
$$

Where, $S_{i}$ is the transparency of the number $i$ layer; $I_{\text {old }}$ is the original light intensity of this pixel, and $I_{\text {new }}$ denotes the light intensity after color blended.

\section{Experimental Results}

The proposed approach is implemented on the platform of Windows 10 system with VC++ and DirectX 11 programs, installed in a computer with Intel Core 15-4440, 8GB memory and NVIDIA GeForce GT 635. DirectX 11 is the mainstream interface of graphic rendering engine and it has the vertex shader, geometry shader and fragment shader needed in the proposed approach.

The test sample is what's shown in Figure 2, which has a repeat size of 154 courses and 112 wales. The mean time consumed for this simulation is 520 milliseconds, which is able to meet the requirement of simulation efficiency. The operations of zooming in/out and rotating are performed via GPU, eliminating the possibility of obvious lag.

The simulation result of technical back is shown in Figure 11-a and its partial, enlarged pattern is shown in Figure 11-b. The corresponding partial, enlarged result of technical face is shown in Figure 11-c. A small tuft of piles gather together on the technical face.

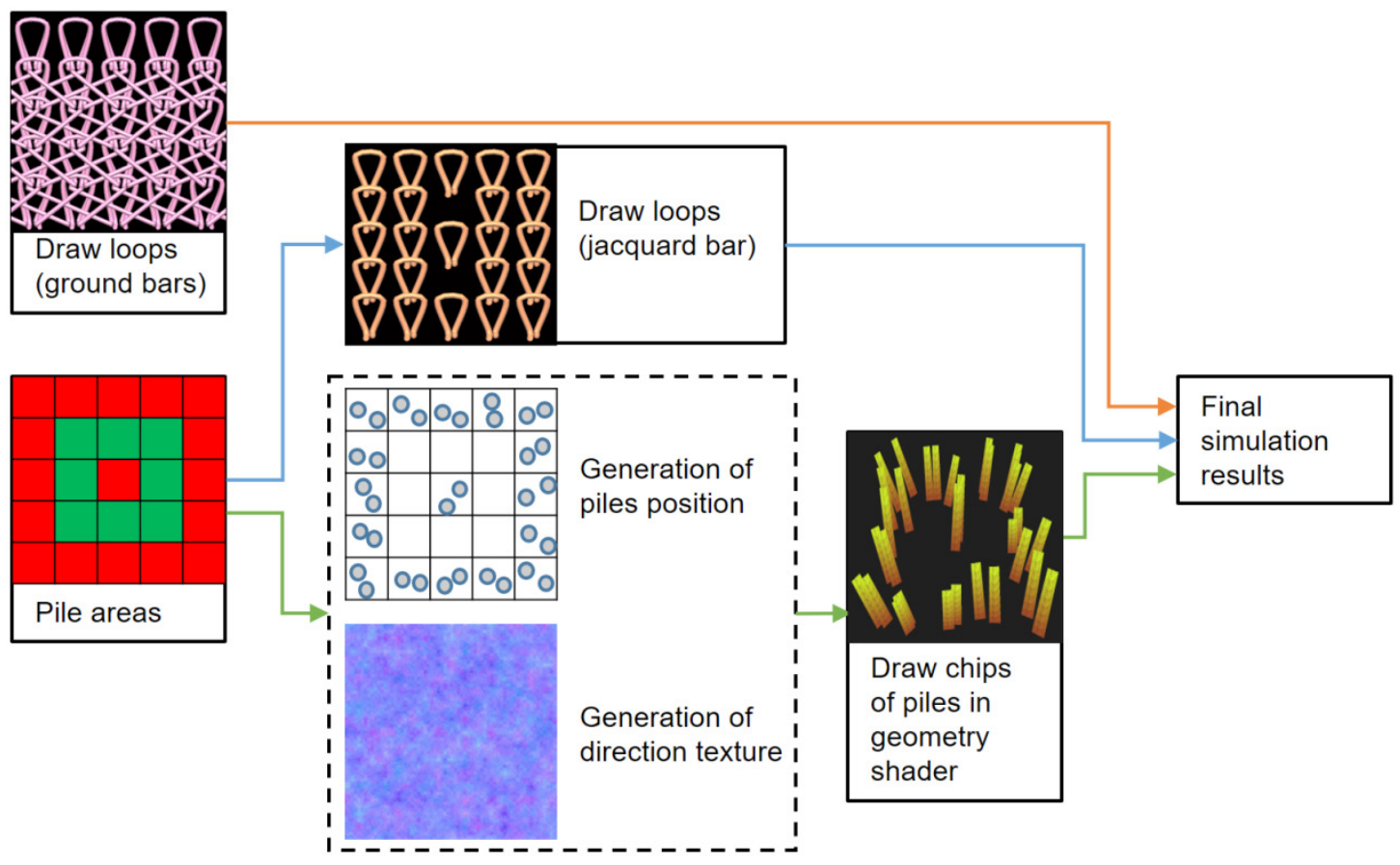

Figure 10. Simulation flowchart of jacquard-patterned plush fabrics 


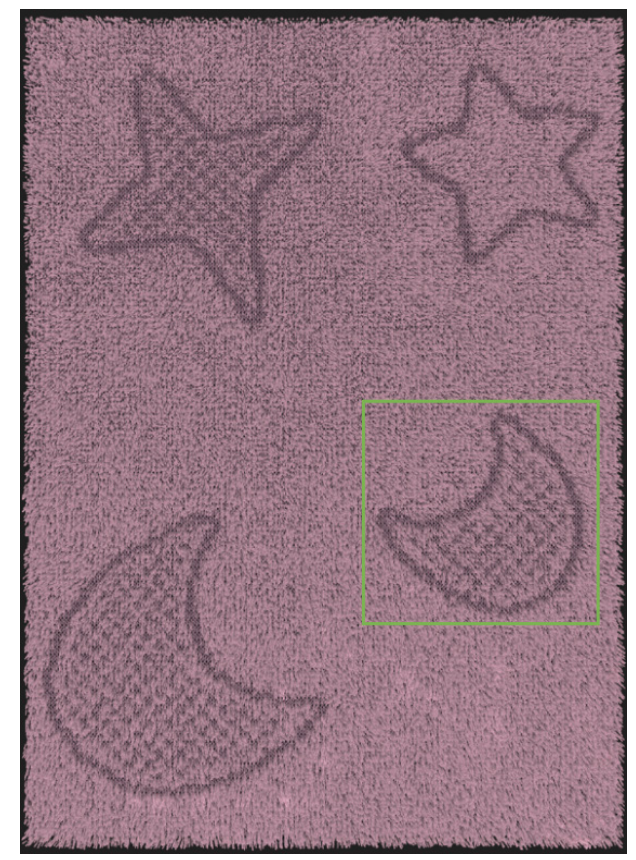

(a)

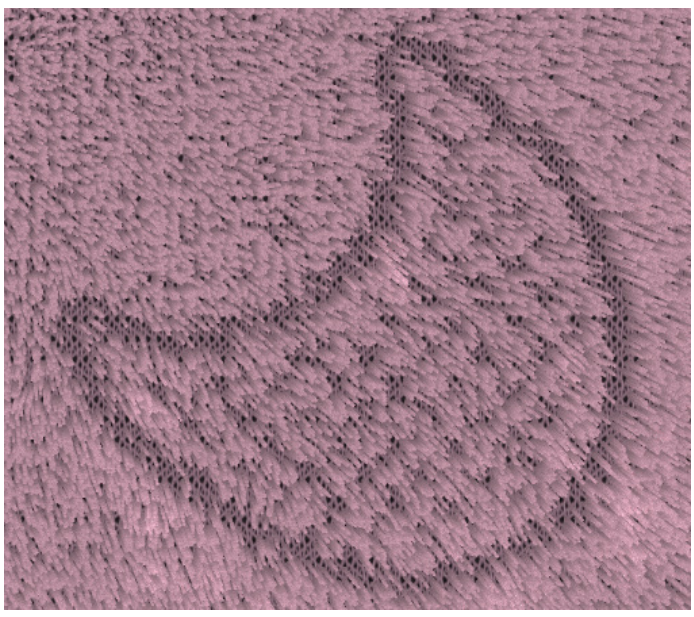

(b)

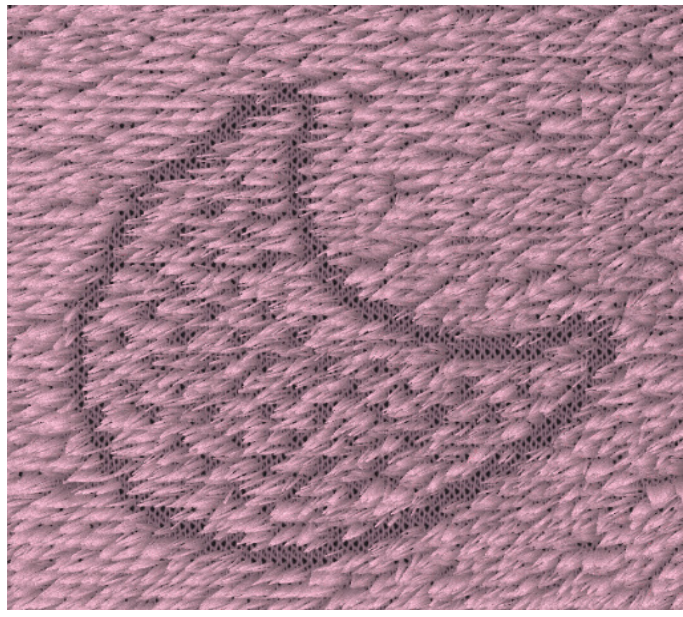

(c)

Figure 11. Simulation results. (a) an overall result on technical back; (b) an enlarged partial result on technical back; (c) an enlarged partial result on technical face.

\section{Conclusion}

The knitting and jacquard principles of double-bar plush fabrics with jacquard patterns are analyzed in this paper. Based on that the analysis, a tailored simulation model is built with the idea of multi-layered texture. It achieves realistic simulation results of pile patterns on both technical face and technical back via geometry shader. Two sorts of texture chips are used in the proposed method to avoid exposing interstice between texture layers when the pile yarn is observed from different directions. Random offsets of pile are achieved by using the Perlin fractal noises to make it more realistic. The experimental results obtained from the simulation model confirm the efficiency and practicability in computer-aided design software.

However, the shadow of each pile is not separately computed in this paper, resulting in limitation when showing coverage relationship among pile yarns. In addition, due to the selection of a single pile yarn as one unit, the simulation result drawn in this paper is less soft and gentle as compared to the one drawn with a region of pile yarns as the unit.

\section{Acknowledgements}

The authors acknowledge the financial support from the National Science Foundation of China (No. 61602212), the Innovation fund project of Cooperation among Industries, Universities \& Research Institutes of Jiangsu Province (No. BY2015019-31), the Fundamental Research Funds for the Central Universities (JUSRP115A02) and A Project Funded by the Priority Academic Program Development of Jiangsu Higher Education Institutions (PAPD, No.[2014]37). 


\section{References}

[1] Cong HL, Li XL. Simulation of jacquard pile fabric based on texture synthesis[J]. Journal of Textile Research, 2014, 35(10):150-155.

[2] Lei $H$, Cong $H L$, Zhang AJ. CAD design and simulation of double-needle bar short pile fabrics[J]. Journal of Textile Research, 2013, 34(7): 132-136.

[3] Xiong Y, Miao XH, Zhang AJ, et al. Computer simulation for warp knitted brushed fabric with patterned pile[J]. Textile Research Journal, 2016, 86(15): 1659-1667.

[4] Csuri C, Hakathorn R, Parent $R$, et al. Towards an interactive high visual complexity animation system[C]. Acm Siggraph Computer Graphics, 1979, 13(2):289-299.

[5] Neulander I. Quick image-based lighting of hair[C]. Acm Siggraph 2004 Sketches. ACM, 2004:43.

[6] Sadeghi I, Pritchett $H$, Jensen $H W$, et al. An artist friendly hair shading system[J]. Acm Transactions on Graphics, 2010, 29(4):157-166.

[7] Yan $L Q$, Tseng CW, Jensen $H W$, et al. Physicallyaccurate fur reflectance[J]. Acm Transactions on Graphics, 2015, 34(6):1-13.

[8] J.T. Kajiya, T.L. Kay. Rendering fur with three dimensional textures[C]. ACM SIGGRAPH Computer Graphics, 1989, 23(3): 271-280.

[9] Lengyel J. Real-time fur[M]. Rendering Techniques 2000. Springer, Vienna, 2000: 243-256.
[10] Lengyel J, Praun E, Finkelstein A, et al. Real-time fur over arbitrary surfaces[C]. Proceedings of the 2001 symposium on Interactive 3D graphics. ACM, 2001: 227-232.

[11] Yang G, Cao WQ, Huang XY. A fur modeling system based on multi-layer textured slices[J]. Journal of Image and Graphics, 2008; 13(5): 984-990.

[12] Tang Y, Shao XQ, Lv MY. Improved Lengye's method based fur real-time simulation[C]. Proceedings of the Second 3-D Image Technology and Application Conference, 2007; 139142.

[13] Wong S, Fu I. Hybrid-based snow simulation and snow rendering with shell textures[J]. Computer Animation \& Virtual Worlds, 2015, 26(3-4):413-421.

[14] Lee J, Kim D K, Kim H S, et al. Real-time fur simulation and rendering[J]. Computer Animation \& Virtual Worlds, 2010, 21(3-4):311-320.

[15] Zhang AJ, LiXX, et al. 3D simulation model of warp-knitted patterned velvet fabric [J]. International Journal of Clothing Science and Technology, 2016, 28(6): 794-804.

[16] Han YM, Miao XH, Yu GQ, et al. Production Equipment and Process Characteristics of Double Needle Bed Warp Knitted Velvet Fabrics[J]. Knitting Industries, 2014(12):1214.

[17]Perlin K. Improving noise[J]. Acm Transactions on Graphics, 2002, 21(3):681-682. 